\title{
Letter From the Editor in Chief, Renee Bittoun
}

\section{Ethics, morals and nicotine}

Smoking cessation has been the primary goal for those of us working in tobacco treatment for many decades. However, we have all noticed that in countries where there is a strong anti-smoking climate, there seems to be an increasing percentage of smokers, willing and motivated to quit, who, despite all efforts on our part, find it very difficult to achieve this. 'Harm reduction' or 'harm minimisation' strategies have become part of our agenda and is now offered routinely to smokers in these circumstances. As these strategies invariably incorporate concurrent smoking with nicotine-containing products, there is the dilemma of on-going nicotine usage, or 'therapeutic/medicinal nicotine' for extended periods of time (though evidently better and safer than smoking alone).

The concern addressed here is that nicotine is not made synthetically and is derived from the tobacco plant. There has long been a link between the tobacco growers and the pharmaceutical companies that make nicotine products such as nicotine replacement therapies (NRT). When our agenda has been cessation, these products have helped us achieve that goal. Our harm reduction strategies, concurrent smoking and NRT have helped smokers and have been a 'gateway' to quitting. However, there are now products entering the market that contain tobacco constituents that are manufactured and marketed directly by the tobacco industry. Snus is one of the best known of current products with openly direct links with the tobacco industry. This is just one of many. There are a myriad of ecigarettes available that contain nicotine and trials have begun to assess their efficacy in both reducing smoking and increasing cessation.

Do we recommend these products? Are they safe? Are they safer than smoking (which seems to be the case). Does using these products prolong dependence and therefore are they as benign as the tobacco industry would like us to believe? Is recommending these products or conducting trials using these products, 'supping with the devil' as some have suggested? Does this give legitimacy to the tobacco industry, who collectively have a notorious history of dissimulation and deception, as well as never having had the public health at heart and have given many of us (myself included) personal legal grief?

The review paper by Rutqvist et al. concerning Snus as a smoking cessation product, in this issue of the Journal of Smoking Cessation, is published in order to openly describe strategies that are being used within the tobacco treatment community. The authors of the review acknowledge direct links with the tobacco industry. This is the first time a paper has been published in this journal with direct links to the tobacco industry.

These strategies may not sit easily within the moral and ethical philosophies we hold, however, this comment has been made so that a debate might commence regarding this matter between those of us who are tobacco treatment specialists.

Letters to the Editor are welcome. 